\title{
FEATURE Leading at the edge: A roadmap to advance edge of field practices in agriculture
}

\author{
Catherine DeLong, Clare Lindahl, and Kris Johnson
}

A nyone who has driven cross-country knows one thing: America's landscape is as diverse as it is beautiful. From the sandy shores of the Atlantic Ocean, rich soils of the Midwest, and flat expanse of the Mississippi River Delta, to the arid deserts of the Southwest and the forests of the Pacific Northwest - the landforms, climates, and resource concerns are different. In order to meet the diverse resource concerns of these landscapes, we need a diverse set of tools from which farmers, ranchers, and landowners can choose.

Although each piece of land is different, we are all interconnected through our shared natural resources; choices on a farm in central Indiana can affect the drinking water of local communities and the livelihoods of fishermen and fisherwomen in the Gulf of Mexico. Looking at this from a national perspective, it is clear that a whole-farm-or whole-systems - approach to agriculture production is necessary to meet water quality, carbon (C) and habitat goals. This means offering farmers and landowners a toolbox of conservation practices to ensure the continued resilience of their land. However, right now, a key part of that opportunity is being missed.

As part of a systems approach to agriculture, farmers, ranchers, and landowners need access to both in-field and edge of field (EoF) conservation practices. To address resource concerns and meet conservation goals, EoF practices are one part of a whole-systems approach that includes infield soil health and nutrient management practices, as well as downstream floodplain protection and restoration efforts.

Edge of field practices are designed to slow, filter, and process both surface runoff (aboveground flow) and subsurface flow (belowground water commonly from tile drainage systems) from farm fields. These practices include vegetated and riparian buffers, filter strips, and restored wetlands, as well as bioreactors, saturated buffers, and constructed wetlands (figure
1). These practices provide water quality benefits, $\mathrm{C}$ and flood water storage, pollinator and wildlife habitat, and streambank stabilization. They are an important form of infrastructure in rural America and a potential source of jobs as they often require engineers, construction crews, and technicians to plan, construct, and manage these projects. Edge of field practices also have the added benefit of being "stackable," meaning they can be paired with in-field conservation practices to support farm productivity and profitability while maximizing the conservation benefits (Christianson et al. 2018).

However, EoF practices have not yet been applied at meaningful scales, likely due to up-front costs, technical barriers, and misperceptions about the role these practices can play on productive, profitable farms. These barriers must be overcome so that EoF practices can be deployed on the landscape to improve water quality, store $\mathrm{C}$, create habitat, and provide other benefits for farmers and their communities.

\section{A ROADMAP TO ADVANCE EDGE OF FIELD PRACTICES}

In an effort to remedy this missed opportunity, The Nature Conservancy (TNC), the Soil and Water Conservation Society (SWCS), and Meridian Institute have recently collaborated to unveil Leading at the Edge: A Roadmap to Advance Edge of Field Practices in Agriculture (EoF Roadmap) (TNC 2021). The EoF Roadmap is a guide for future actions, highlighting both bottlenecks and opportunities for growth, and serves as a blueprint for collaboratively scaling up EoF adoption.

Over the last year, TNC, SWCS and Meridian Institute, with support from the Walmart Foundation, convened 26 partners with expertise and experience in agriculture, conservation, water issues, and relevant research, policy, and practice. Partners included farmer leaders and early adopters of EoF practices; representatives from environmental and conservation nonprofits, commodity groups, professional associations, and agriculture and food companies; and current and former public agency staff. This diverse group collaborated to develop a cohesive set of recommendations that, if implemented together, will help achieve shared goals for improved water quality, enhanced habitat, $\mathrm{C}$ sequestration, and resilient working lands and communities.

The EoF Roadmap contains nine recommendations (figure 2) hinging on three pillars of action: build the economic case, increase technical assistance and capacity, and elevate a culture of conservation and innovation in agriculture. It also includes a comprehensive literature review on the science surrounding these practices, including their benefits, opportunities for future research, and cost-effectiveness relative to other interventions. The EoF Roadmap takes a holistic look at the opportunities to scale up EoF practices, including opportunities within markets, conservation finance, and green infrastructure. It features successful and innovative case studies from across the nation and acknowledges the accomplishments of individuals and organizations, as well as the progress that has already been made in advancing these practices.

The EoF Roadmap is part of a theory of change that identifies state, regional, and national policy options, evaluates those options through science-informed criteria, and defines the key science, economic, and policy levers to drive EoF practice implementation to scale. It compliments an existing Soil Health Roadmap (TNC n.d.) and uniquely articulates EoF implementation barriers for TNC and partners to collectively pursue. When combined with soil health and downstream practices, EoF practices

Catherine DeLong is special projects and policy director and Clare Lindahl is chief executive officer of the Soil and Water Conservation Society, Ankeny, lowa. Kris Johnson is interim director of agriculture, North America, The Nature Conservancy, Minneapolis, Minnesota.

Received February 26, 2021. 


\section{Figure 1}

Examples of edge of field practices (TNC 2021).

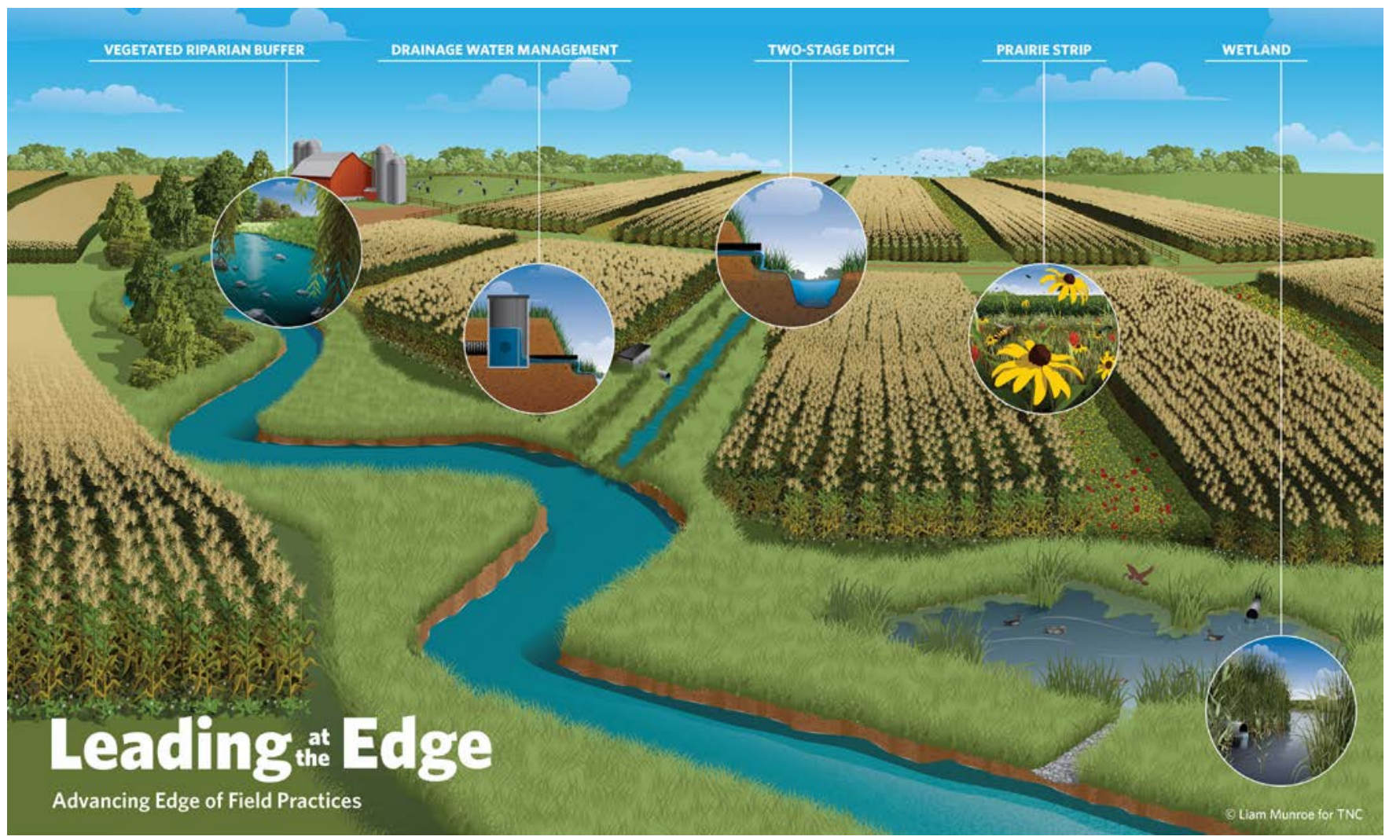

are an essential part of a whole-systems approach to achieve prosperous farms, clean water, and resilient communities.

\section{GROUNDWORK FOR EDGE OF FIELD PRACTICE ADVANCEMENTS}

SWCS has been working to expand the science and adoption of EoF practices for nearly a decade. Early on, SWCS recognized the potential of these practices to reduce nutrient runoff and leaching to our waterways and broaden the network of stakeholders contributing to conservation. SWCS's initiatives included hosting the Nutrient Management and Edge of Field Monitoring Conference in 2015, which convened over 200 researchers, conservation professionals, and farmers from across the nation to discuss EoF monitoring research, nutrient reduction strategies and activities, and innovative conservation programs. A 2018 special issue of the Journal of Soil and Water Conservation followed the conference, titled "Edge-of-Field Monitoring for Nutrient Losses" (vol-

\section{Figure 2}

Summary of Edge of Field Roadmap recommendations (TNC 2021).

\section{RECOMMENDATIONS}

1. Elevate and replicate successful or promising local, state, and regional policies and initiatives.

2. Remove administrative barriers to conservation practice implementation.

3. Increase technical assistance by supporting the multiple sources of conservation expertise.

4. Integrate EoF as a nature-based water management policy solution.

5. Increase funding and better target conservation programs to achieve watershed-scale impacts.

6. Expand innovative funding approaches, like ecosystem services markets.

7. Accelerate sustainable supply chains and corporate commitments to water and biodiversity.

8. Harness and extend efforts to rebuild soil health to recognize the vital role of EoF in working landscapes.

9. Harmonize and coordinate national agriculture policy. 
ume 37, number 1). Over 50 university researchers and USDA scientists collaborated for two years to produce 13 feature and research articles on EoF monitoring. The effort summarized the state of EoF science, adoption, and policy, and offered a roadmap to increase capacity. Most recently, SWCS has worked to advance the knowledge and branding of EoF practices by including saturated buffers, bioreactors, drainage water management, prairie strips, and (coming in 2021) wetlands in our Conservation Media Library, an open-source multimedia storing house of conservation photos, videos, graphics, etc. for farmers, conservation professionals, and the media.

SWCS has also led and collaborated on on-the-ground projects to scale up water quality wetlands in Iowa by identifying opportunities for funding, cost-saving, and speeding up the timeline from farmer interest to project completion. Today, SWCS continues its leadership to enhance the current model for EoF adoption by creating practice process models, identifying inefficiencies in practice design and implementation, improving communication, expanding partnerships, and engaging leadership necessary to scale up EoF practices.

As a co-convener of the EoF Roadmap, and as the organization that represents and supports conservation professionals across the globe, SWCS is excited to heed the roadmap's call to action. We hope that conservation professionals will find it useful and inspiring, and will be able to see their work reflected in its recommendations.

For TNC, EoF practices are a key part of the solution to advance regenerative agriculture, which includes strategic partnerships to achieve our conservation goals. A science-based conservation organization founded nearly 70 years ago, TNC's mission is to conserve the lands and waters on which all life depends. One key place where the TNC mission touches down is on US working lands. We are working with the agricultural community to advance regenerative practices that build soil health and increase resilience and profitability for farmers and ranchers. These are the very practices that enable the agriculture industry to play a key role in restoring habitat, improving water quality, and combatting climate change.

\section{CALL TO ACTION}

EoF practices offer one more tool in the toolbox to reach our water quality, $\mathrm{C}$, and habitat goals. The EoF Roadmap gives agricultural stakeholders and conservation professionals the opportunity to coalesce around a shared vision for collaboration, engagement, and scaling up of EoF practices. Although the landforms, climates, and resource concerns across the nation may vary, we can only achieve meaningful impacts on our natural resources if we work together. Creating the EoF Roadmap is just the beginning; its true value will only be realized through collective action to enact its recommendations.

\section{REFERENCES}

\footnotetext{
Christianson, R., L. Christianson, C. Wong, M. Helmers, G. McIsaac, D. Mulla, and M. McDonald. 2018. Beyond the nutrient strategies: Common ground to accelerate water quality improvemen in the Upper Midwest. Journal of Environmental Management 206:1072-1080

TNC (The Nature Conservancy). n.d. Rethinking Soil, Investing in Our Foundations. https://www. nature.org/en-us/what-we-do/our-insights/ perspectives/rethinking-soil-reinvesting-in-ourfoundations $/$ ? vu=r.v_rethinksoil.

TNC. 2021. Edge of Field Practices in Agriculture. https://www.nature.org/en-us/ what-we-do/our-priorities/provide-food-andwater-sustainably/food-and-water-stories/ farming-for-water-wildlife/.
} 